\title{
A new Hi catalog of Low Surface Brightness galaxies out to $z=0.1$
}

\section{Tripling the number of massive LSB galaxies known $\star, \star \star$}

\author{
K. O’Neil ${ }^{1, \star \star \star}$, G. Bothun ${ }^{2}$, W. van Driel ${ }^{3}$, and D. Monnier Ragaigne ${ }^{3}$ \\ 1 NRAO, PO Box 2, Green Bank WV 24944, USA \\ e-mail: koneil@nrao.edu \\ 2 University of Oregon, Physics Department, Eugene OR, 97402, USA \\ 3 Observatoire de Paris, Section de Meudon GEPI, CNRS UMR 8111 and Université de Paris 7, 5 place Jules Janssen, \\ 92195 Meudon Cedex, France
}

Received 16 January 2004 / Accepted 21 June 2004

\begin{abstract}
Using both the Arecibo $305 \mathrm{~m}$ and the Nançay decimetric 100-m class radio telescopes, we have observed the $\mathrm{H}$ I line of 116 Low Surface Brightness (LSB) galaxies from the Bothun et al. (1985) subset of LSB galaxies in the Uppsala General Catalog. The observations had a detection rate of $70 \%$, resulting in the new determination of HI properties for 81 galaxies. Surprisingly, roughly half of the detected objects (38) have $M_{\mathrm{HI}} \geq 10^{10} M_{\odot}$, placing them into the category of massive LSB galaxies. As previously only $\sim 18$ of these "Malin 1 cousins" were known, our results have more than tripled the number of these fascinating and enigmatic systems known.

Combining our results with previous studies done on the Bothun et al. catalog results in a well-defined catalog of H I properties of $526 \mathrm{LSB}$ galaxies ranging in redshift space from $0 \leq z \leq 0.1$. With this catalog in hand, we have been able to explore the parameter space occupied by LSB galaxies more completely than has been previously possible. In agreement with previous studies, our results show LSB galaxies with some of the most extreme properties of disk galaxies, including $M_{\mathrm{HI}} / L_{\mathrm{B}}$ ratios often exceeding $10 M_{\odot} / L_{\odot, \mathrm{B}}$.
\end{abstract}

Key words. galaxies: distances and redshifts - galaxies: spiral - galaxies: luminosity function, mass function galaxies: fundamental parameters

\section{Introduction}

Low Surface Brightness (LSB) galaxies, those objects with central surface brightness at least one magnitude fainter than the night sky, are now well established as a real class of galaxies with properties distinct from those that define the Hubble Sequence (e.g., Impey \& Bothun 1997; Bothun et al. 1997, and references therein). Yet considerable uncertainty still exists as to both the range in properties of these galaxies and their number density in the $z \leq 0.1$ Universe. As LSB galaxies encompass many of the "extremes" in galaxy properties, gaining a firm understanding of LSB galaxy properties and number counts is vital for testing galaxy formation and evolution theories. Additionally, in this era of precision cosmology

* Tables 1-3 are only available in electronic form at the CDS via anonymous ftp to cdsarc.u-strasbg.fr $(130.79 .128 .5)$ or via http: //cdsweb.u-strasbg.fr/cgi-bin/qcat?J/A+A/428/823

$\star \star$ Appendix is only available in electronic form at http://www. edpsciences.org

$\star \star \star$ Work done while at Arecibo Observatory. (e.g. Spergel et al. 2003) it becomes increasingly important to determine the relative amounts of baryons that are contained in galaxy potentials compared to those that may comprise the Intergalactic Medium. As long as the possibility exists that optical and baryonic luminosity functions are significantly biased toward galaxies with relatively high surface brightness, we will not be able to obtain a clear understanding of the percentage of baryons which lie in galaxies.

The "traditional" perception of LSB galaxies is that they are low mass, fairly blue systems with relatively high $M_{\mathrm{HI}} / L_{\mathrm{B}}$ values and low metallicities (Bergmann et al. 2003; Bell et al. 1999; Gerritsen \& de Blok 1999; de Blok et al. 1995). Consequently, LSB galaxies are often equated with young dwarf galaxies (Dekel \& Woo 2003; Cabanela \& Dickey 2002; Jimenez et al. 1998). However, the simple, consistent, and repeatable observation that LSB disk galaxies can be found at any value of circular velocity (or total mass) is often under-appreciated, reinforcing the erroneous perception that LSB galaxies are strictly low mass systems. The current set 
of observations for all LSB galaxies shows them to have a remarkably diverse array of properties:

- very red LSB galaxies have been found by optical surveys searching in both the $B$ and $V$ optical bands (Burkholder et al. 2001; O'Neil et al. 1997);

- recent H I surveys by, e.g. Burkholder et al. (2001) and O'Neil et al. (2000) show no tendency toward LSB systems having lower than average H I masses and/or total dynamical masses. The LSB sources with H I detections in Burkholder et al. (2001), for example, have on average $\left\langle\log \left(M_{\mathrm{HI}} / M_{\odot}\right)\right\rangle=9.0 \pm 0.6$, compared to $\left\langle\log \left(M_{\mathrm{HI}} / M_{\odot}\right)\right\rangle=$ $9.4 \pm 0.6$ for that survey's High Surface Brightness (HSB) galaxy sample - both samples have the same mean and range of values of circular velocity;

- a number of massive $\left(M_{\mathrm{HI}} \geq 10^{10} M_{\odot}\right)$ LSB systems have been found, including Malin 1 - the largest disk galaxy known to date (Matthews et al. 2001; Pickering et al. 1997; Davies et al. 1988; Sprayberry et al. 1995);

- although the average LSB galaxy does have lower metallicities than the average HSB galaxy, LSB galaxies with near-solar abundances have been found (Bell 2000);

- while many LSB systems with extremely high $M_{\mathrm{HI}} / L_{\mathrm{B}}$ values have been found, the argument that $\left.\left\langle M_{\mathrm{HI}} / L_{\mathrm{B}}\right\rangle_{\mathrm{LSB}}\right\rangle$ $\left\langle M_{\mathrm{HI}} / L_{\mathrm{B}}\right\rangle_{\mathrm{HSB}}$ may be due more to the nature of the H I LSB galaxy surveys - since redshifts for LSB systems are often found by searching in H I, LSB galaxies without detectable quantities of $\mathrm{H}$ I do not make it into databases, artificially raising the value of $\left\langle M_{\mathrm{HI}} / L_{\mathrm{B}}\right\rangle_{\mathrm{LSB}}$. Countering this trend, the recent survey of Burkholder et al. (2001) obtained optical redshifts for a large sample of LSB galaxies which were also observed in $\mathrm{HI}$. The resultant catalog contains LSB systems with $M_{\mathrm{HI}} / L_{\mathrm{B}}$ as low as $10^{-6} M_{\odot} / L_{\odot, \mathrm{B}}$.

While none of the above results contradict the idea that the $a v$ erage LSB galaxy is less evolved than the average HSB galaxy, they do show that we have not yet come close to fully sampling the LSB galaxy parameter space. In addition, it should be emphasized that there may still be large numbers of LSB galaxies with properties beyond our present detection limits (e.g., Sabatini et al. 2003).

One issue related to the study of LSB galaxies lies in their potential contribution to the total baryonic content of the Universe. A wide variety of data and opinions have been offered on this topic. Blind HI surveys, such as those done by Kilborn et al. (2002), Rosenberg \& Schneider (2000), Kraan-Korteweg et al. (1999), Knezek (1999) and Zwaan et al. (1997) detect no massive LSB galaxies in the local $(z \lesssim 0.025)$ Universe, leading to the conclusion that LSB galaxies could not be significant contributors to the local H I mass function (HIMF) and subsequently to the baryonic mass function. Similar conclusions have also been reached based on optical surveys (e.g., Blanton et al. 2003; Cross et al. 2001). Such a conclusion, however, is valid only if one believes that a fair and representative volume of the Universe has been probed in both the optical and $21-\mathrm{cm}$ bands, and that the properties of the objects found in these surveys is well understood.

The analysis of the WMAP results have provided a precision measure of the total baryonic content of the Universe, albeit in a model dependent way. In normalized units, the contribution of baryons to the total energy density of the Universe is $4.5 \%$. This contribution is 12 times higher than that which is contained in optically selected catalogs of galaxies which do not contain LSB galaxies (Shull 2003; Bothun 2003) which either means that a) we are missing most of the galaxy population in our current catalogs; or b) $95 \%$ of the baryonic content of the Universe is in the (presumably warm) IGM. At present, the needed baryons in the warm IGM (alternative b) remain undetected. Moreover, the best fitting 6-parameter WMAP model contains a curious result - the global ratio of baryonic energy density to dark matter energy density is 0.19 . This is two times larger than the baryonic mass fraction that is typically found in disk galaxies from analysis of rotation curves and clearly implies the existence of undetected baryons. It is possible that the undetected population is LSB galaxies.

It has been repeatedly shown that the question of how much a galaxy type contributes to the baryon (and matter) density in the Universe is twofold - what is the number density of massive galaxies of that type, and what is the slope of the low mass luminosity/baryon density function. Whereas numerous surveys are (and have been) undertaken to address the latter question (e.g., Sabatini et al. 2003; Mobasher et al. 2003), here we aim to look into the former. In a nutshell, we would like to examine the questions "how many massive LSB disk galaxies are in the $z \leq 0.1$ Universe, and what are their properties?"

To shed light on these questions, we have re-investigated one of the first surveys designed to measure the space density of LSB galaxies. Bothun et al. (1985) measured H I masses and redshifts for a well-defined sample of LSB galaxies chosen from the UGC (Nilson 1973). While that survey was reasonably successful, there were a significant number of $21-\mathrm{cm}$ non-detections which indicated the presence of either a gas poor population of LSB disks or large LSB disks beyond the redshift sensitivity limit of the survey (about $12000 \mathrm{~km} \mathrm{~s}^{-1}$ ). The primary impetus behind our re-investigation, then, was the upgrade of the $305 \mathrm{~m}$ Arecibo telescope. With its improved sensitivity, baselines, and spectrometer, the Arecibo telescope is able to cover a much larger region of velocity space with higher sensitivity than has previously been achievable at $21-\mathrm{cm}$. Consequently, we chose re-examine Bothun et al.'s original H I catalog, compiling all objects with published H I properties (both those detected in the original catalogs and those which were not) and re-observing the objects which were originally not detected. The end result is a well-defined catalog of $\mathrm{H} \mathrm{I}$ properties of $526 \mathrm{LSB}$ galaxies ranging in redshift space from $0 \leq z<0.1$. With this catalog in hand, we are more fully able to explore the parameter space occupied by LSB galaxies.

\section{Catalog selection}

The objects chosen for observation were taken from the sample of LSB galaxies selected from the UGC by Bothun et al. (1985), which meet the following criteria:

- morphological type (as given in UGC) of Sb or later;

- blue surface brightness of $\langle\mu\rangle=m_{\mathrm{pg}}+5 \log (D)+$ $8.63>25.0 \mathrm{mag} \operatorname{arcsec}^{-2}$ (where $m_{\mathrm{pg}}$ is the photographic 
magnitude from the UGC, $D$ is the diameter in arcmin, and 8.63 is the conversion to a $\left(D^{2}\right)$ circular aperture in arcsec). This definition, used by Bothun et al. (1985), is typically equivalent to the more commonly used classification of a galaxy as LSB if it has $\mu_{\mathrm{B}, 0}^{\text {observed }} \geq 23.0 \mathrm{mag} \operatorname{arcsec}^{-2}$, although the uncertainty of this equation, combined with the high error of the UGC magnitudes $( \pm 0.5)$ means this catalog may contain objects with central surface brightnesses as high as $\mu_{\mathrm{B}, 0}=22 \mathrm{mag} \operatorname{arcsec}^{-2}$;

- declination in the range $0^{\circ}-36^{\circ}$.

A total of 1865 galaxies meet the above criteria. Of these, 571 were randomly selected and observed by Bothun et al. (1985) with 334 being detected (and later confirmed) in H I. In the intervening years, 107 of the galaxies not originally detected by Bothun et al. have had their H I properties published in the literature. The remaining 130 objects break down as follows:

- 4 galaxies were rejected from the catalog due to being part of an interacting group (UGC 3737, UGC 11564, UGC 11027, and UGC 11057);

- 15 of the objects listed as detections by Bothun et al. (1985) were not confirmed by follow-up observations and are considered non-detections for this paper;

- 86 galaxies from the original list were observed for this paper;

- the remaining 25 galaxies, all of which were listed as nondetections by Bothun et al. (1985) were not observed for this paper (due to scheduling restrictions), and have had no other follow-up H I observations.

To compensate for the "missing" galaxies, an additional 24 galaxies, randomly chosen from the original catalog of 1865 objects, were observed. Additionally, 6 LSB galaxies from the catalog of O'Neil et al. (1997) which fit into the scheduled observing time on the Arecibo telescope were also observed. The final result is a catalog of $561 \mathrm{HI} \mathrm{ob-}$ servations of LSB galaxies, 116 of which are new observations. A complete list of the 116 newly observed galaxies and their optical properties is given in Tables 1 and 2 . The HI and optical properties of all other galaxies, both those originally detected by Bothun et al. (1985) and those later detected in the literature, can be found on-line at http://www.gb.nrao.edu/ koneil/data.

\section{Observations}

For efficiency, observations were made using both the Arecibo $305 \mathrm{~m}$ and the 100-m class Nançay radio telescopes. Details of observations with the individual telescopes are given below, published properties of the galaxies are given in Table 1 , and a complete listing of the observations is given in Table 2 .

\subsection{Arecibo observations}

Observations with the Arecibo $305 \mathrm{~m}$ telescope were taken between 16 May, 1999-2 April, 2002. Data was taken using the L-wide and L-narrow receivers and two separate polarization channels. Two different correlator set-ups were used - one for galaxies with published velocities and one for galaxies whose velocity was unpublished at the time of the observations. The objects without published velocity were observed using four $50 \mathrm{MHz}$ band passes with 2048 lags and 3-level sampling, giving an unsmoothed resolution of $5.2 \mathrm{~km} \mathrm{~s}^{-1}$ at $1420 \mathrm{MHz}$. The frequency range covered for these observations was either $1270-1425 \mathrm{MHz}$ or $1238-1423 \mathrm{MHz}$. (Two different frequency ranges were used due to the increasingly frequent presence of a strong radar at $\sim 1240-1260 \mathrm{MHz}$.) In both cases sufficient overlap was given to the individual correlator boards to effectively eliminate any problems which otherwise may have arisen due to poor performance in the outer 50 channels of each correlator.

If an observed galaxy already had a published velocity, only two correlator boards were used. In this case each board was centered at the galaxy's redshifted H I line, recorded a separate polarization channel, and had a total of 2048 lags across its 12.5 MHz band. Here, all the data had 9-level sampling.

All Arecibo data was observed in position-switched mode, with a minimum of one $10 \mathrm{~min}$ on- and off-source observation pair taken of each galaxy, followed by a $20 \mathrm{~s}$ ON/OFF observation of a calibrated noise diode. In most cases a galaxy was observed at least twice using this method, and often as many as 4-6 times. System temperatures were obtained from the noise diode observations, using the predetermined temperature values from Arecibo (available on-line at http://www.naic.edu). A gain curve for the telescope was obtained through reducing all observations taken of standard continuum calibrators, by any project, during the observing period. A complete description of the procedures used to determine the gain curve in this manner can be found in Heiles (2001). Additionally, observations were made of standard continuum calibrators every $2-3 \mathrm{~h}$ during the project observations, with the results checked against the determined telescope gain, to insure no anomalous behavior occurred in the hardware during observations. As a result we can confidently state that the calibration corrections are good to within $10 \%$ (and often much better). A thorough discussion of the errors involved can be found in O'Neil (2004).

\subsection{Nançay observations}

The Nançay decimetric radio telescope, a meridian transit-type instrument of the Kraus/Ohio State design, consists of a fixed spherical mirror (300 $\mathrm{m}$ long and $35 \mathrm{~m}$ high), a tilt able flat mirror $(200 \times 40 \mathrm{~m})$, and a focal carriage moving along a curved rail track. Sources on the celestial equator can be tracked for approximately $60 \mathrm{~min}$. The telescope's collecting area is about $7000 \mathrm{~m}^{2}$ (equivalent to a 94-m diameter parabolic dish). Due to the E-W elongated shape of the mirrors, some of the instrument's characteristics depend on the declination at which one observes. At 21-cm wavelength the telescope's half-power beam width $(H P B W)$ is 3.5 in right ascension, independent of declination, while in the north-south direction it is $23^{\prime}$ for declinations up to $\sim 20^{\circ}$, rising to $25^{\prime}$ at $\delta=40^{\circ}$ (see also 

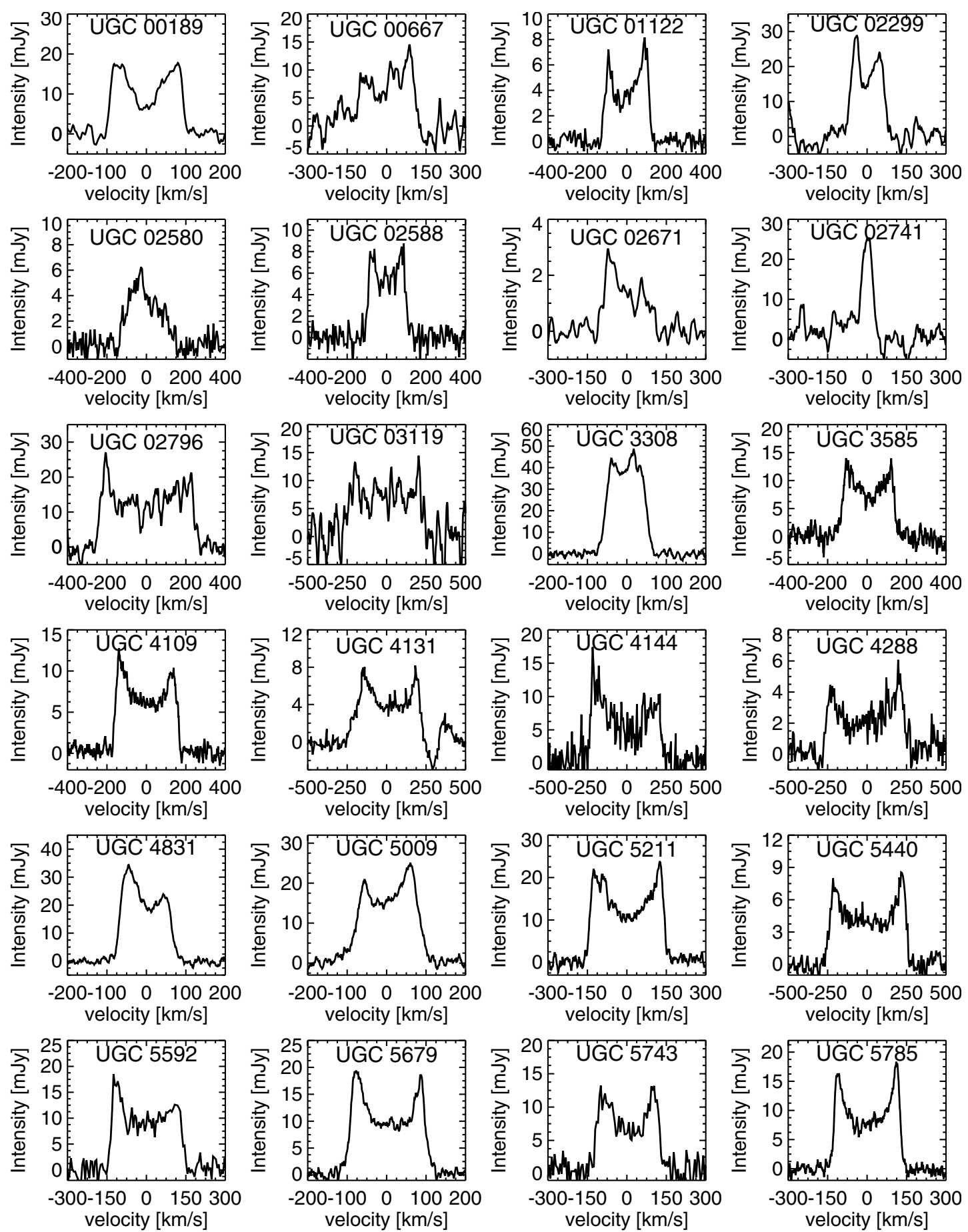

Fig. 1. H I spectra of all detected galaxies, excluding cases of possible confusion. Channel resolution is given in Table 2 . The velocity scale is heliocentric and according to the conventional optical definition.

Matthews \& van Driel 2000). Although the instrument's effective collecting area and, consequently, its gain, follow the same geometric effect, decreasing correspondingly with declination, this effect is negligible for the declination range of the objects in our sample. All observations for our project were made after a major renovation of the instrument's focal system (e.g., van Driel et al. 1997) which resulted in a typical system temperature of $35 \mathrm{~K}$.

The observations were made in the period of JanuaryDecember 2002. using a total of $59 \mathrm{~h}$ of telescope time.
We obtained our observations in total power (positionswitching) mode using consecutive pairs of $40 \mathrm{~s}$ on and $40 \mathrm{~s}$ off-source integrations. Off-source integrations were taken at a position about $20^{\prime} \mathrm{E}$ of the target position.

Only galaxies with published redshifts were observed at Nançay. For all observations the autocorrelator was divided into 1 pair of cross-polarized receiver banks, each with 4096 channels and a $25 \mathrm{MHz}$ bandpass, resulting in a channel spacing of $1.3 \mathrm{~km} \mathrm{~s}^{-1}$. The center frequencies of the 2 banks were tuned to the redshifted HI line frequency of the target 

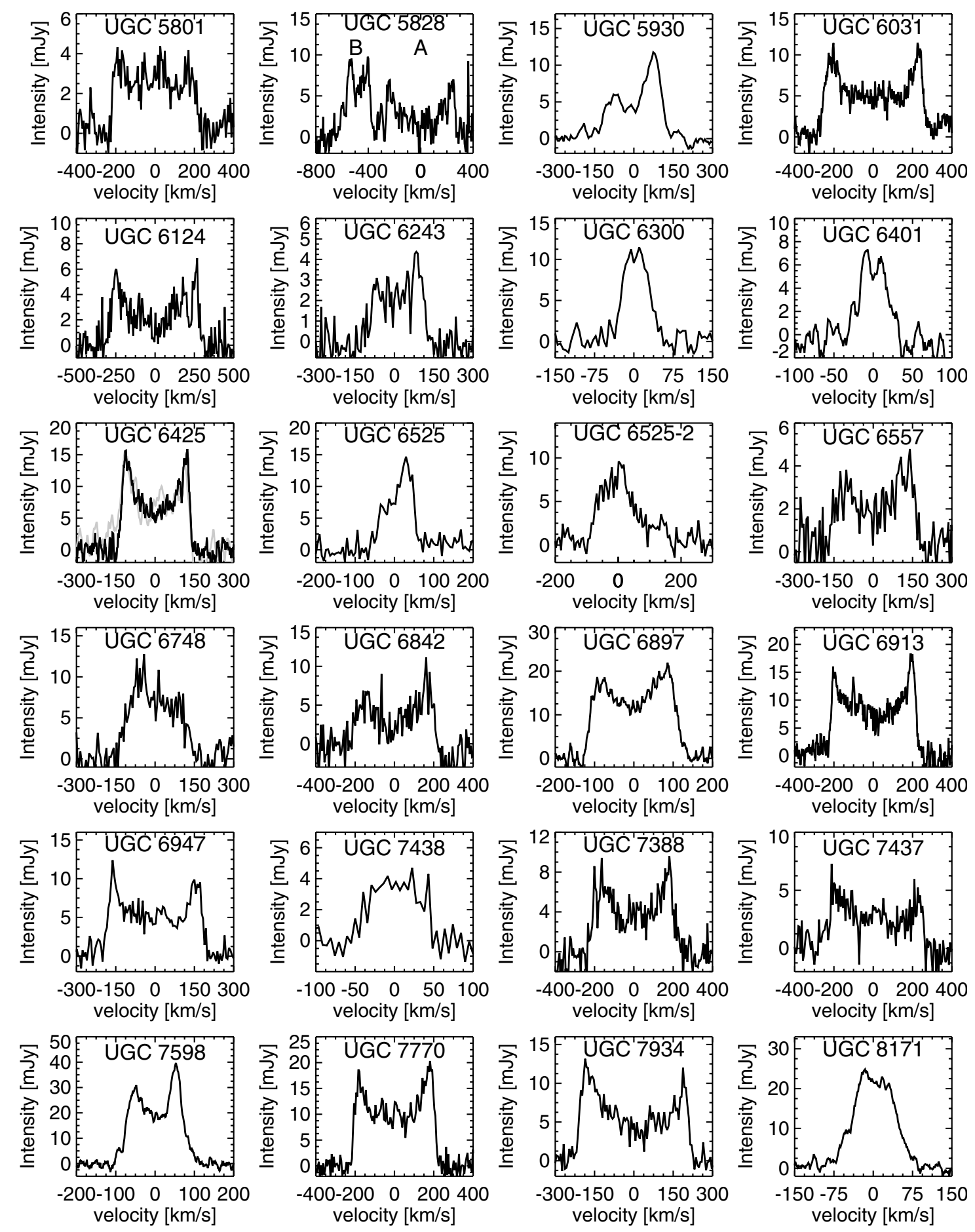

Fig. 1. continued.

source. These spectra were boxcar smoothed to a channel separation of $17.1 \mathrm{~km} \mathrm{~s}^{-1}$ during the data reduction in order to increase signal-to-noise.

Flux calibration, i.e., the conversion of observed system temperatures to flux densities in mJy, is determined for the Nançay telescope through regular measurements of a cold load calibrator and periodic monitoring of strong continuum sources by the Nançay staff. Standard calibration procedures include correction for the above mentioned declination-dependent gain variations of the telescope (e.g., Fouqué et al. 1990). Additionally, a number of standard calibrator galaxies were observed throughout our observing runs, showing the gain used to be within $10 \%$.

In order to reduce the effect of radio frequency interference (RFI) in our observations, we used an off-line RFI mitigation program, which is part of the standard NAPS software package, see Monnier Ragaigne et al. (2003) for further details. It should be noted, however, that not all unwanted emissions 

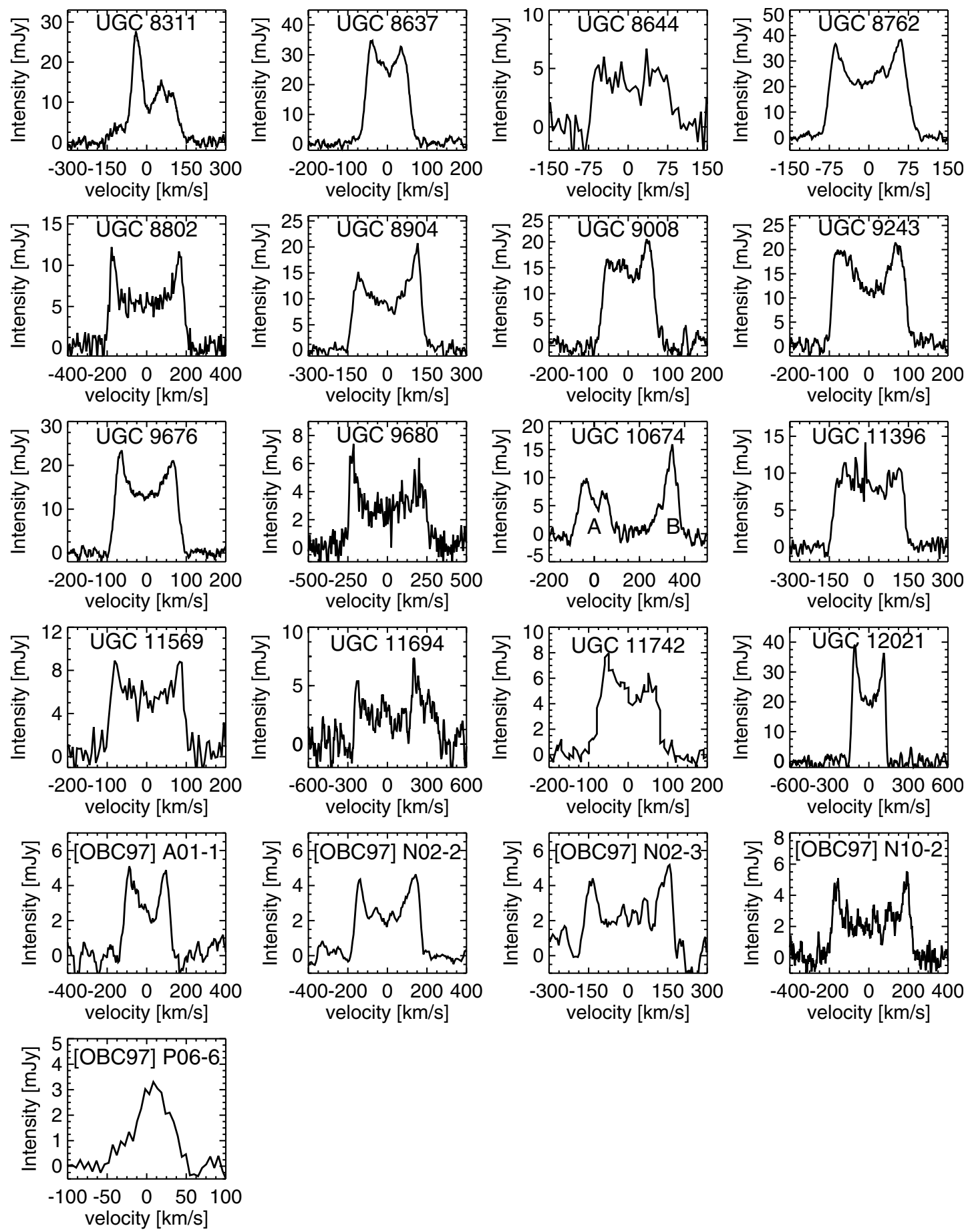

Fig. 1. continued.

could be elimination using this method and that residual signals frequently occur around 4700, 8300, 9500, 11 300, 12700 and $13600 \mathrm{~km} \mathrm{~s}^{-1}$ (Monnier-Ragaigne et al. 2003).

\section{Observational results}

Results of the Arecibo and Nançay observations are given in Tables 2 and 3 and the spectra of all detected galaxies are given in Figs. 1 and 2. Notes on the individual galaxies can be found in Appendix A.
Table 2 lists all galaxies observed, as well as the search range, resolution, telescope used, RFI encountered, and the rms of the RFI-free regions of the spectra. Table 3 lists all observed galaxies which were detected in HI. Table 3 is laid out as follows:

- Col. 1: the galaxy name;

- Col. 2: the heliocentric velocity as measured by our observations; 

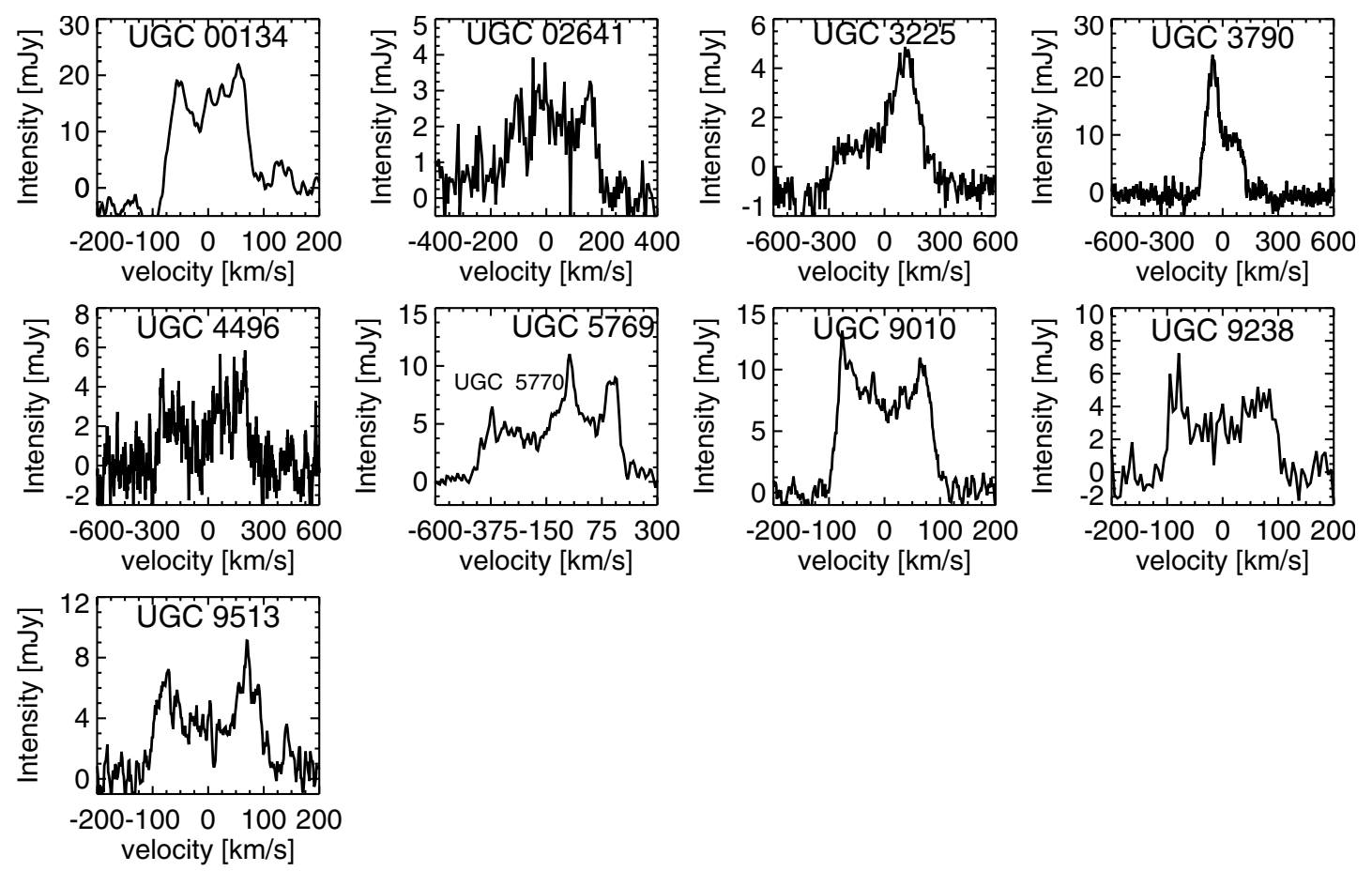

Fig. 2. H I spectra of all detected galaxies which may suffer from confusion. Channel resolution is given in Table 2.

- Cols. 3 and 4: the uncorrected velocity widths at $20 \%$ and $50 \%$ of the peak or average height of the two peaks (when applicable);

- Col. 5: the total measured H I line flux of the galaxy;

- Col. 6: the total HI mass of the galaxy, assuming $H_{0}=$ $75 \mathrm{~km} \mathrm{~s}^{-1} \mathrm{Mpc}^{-1}$ and obtained using the standard formula $M_{\mathrm{HI}} / M_{\odot}=2.356 \times 10^{5}\left(\frac{v\left[\mathrm{~km} \mathrm{~s}^{-1}\right]}{H_{0}\left[\mathrm{~km} \mathrm{~s}^{-1} \mathrm{Mpc}^{-1}\right]}\right)^{2} \int S_{v}[\mathrm{Jy}] \mathrm{d} v ;$

- Col. 7: all previously measured velocities of the galaxy, as found in the literature;

- Col. 8: any notes on the observation regarding potential confusion with other sources. Notes are given in Appendix A.

Figures 10 and 11 show the distribution of the HI mass and corrected H I line width for the objects in the survey against distance. For comparison, the plots also included all other massive LSB galaxies found in the literature, as well as three other Arecibo H I surveys - the "blind" H I surveys of Zwaan et al. (1997) and Rosenberg \& Schneider (2000), and a similar survey to look for high surface brightness CGCG galaxies done by Giovanelli \& Haynes (1993).

\section{5. $\mathrm{HI}$ and stars in LSB galaxies}

Figures 3-9 show the distribution of the $\mathrm{H}$ I and optical properties of the galaxies in the Bothun et al. (1985) catalog for which we have new measurements. From these figures it is clear that the dynamic range of physical properties of LSB disks is large. That is, the UGC LSB galaxy catalog described herein has objects with $\mathrm{HI}$ masses ranging from $6 \leq \log \left(M_{\mathrm{HI}} / M_{\odot}\right) \leq 11$, observed velocity widths of $55 \leq W_{20} \leq 638 \mathrm{~km} \mathrm{~s}^{-1}(58 \leq$ $W_{20}^{\text {cor }} \leq 1276$ ), and $\mathrm{HI}$ mass-to-luminosity ratio within the range $0.04 \leq M_{\mathrm{HI}} / L_{\mathrm{B}} \leq 44 M_{\odot} / L_{\odot, \mathrm{B}}$. Thus, we have a catalog of LSB disk galaxies which range from dwarf through massive galaxies, and include objects which are both gas-rich and gas-poor.

Examining Figs. 3-6 it is clear that while our UGC LSB galaxy sample has similar gas and dynamical masses to that found with HSB galaxies, the $M_{\mathrm{HI}} / L_{\mathrm{B}}$ ratios in the LSB galaxies of this survey are much higher than in typical HSB galaxies. More specifically, the median H I mass of the galaxies in this survey is $4 \times 10^{9} M_{\odot}$, close to the median of $3 \times 10^{9} M_{\odot}$ found in a similar survey of HSB galaxies from the Catalog of Galaxies and Clusters of Galaxies (CGCG) (Zwicky et al. 1961) done by Giovanelli \& Haynes (1993). The dynamical masses are also similar, with $\left\langle M_{\text {dyn }}\right\rangle_{\text {median }}^{\mathrm{LSB}}=3 \times 10^{11}$ and $\left\langle M_{\text {dyn }}\right\rangle_{\text {median }}^{\mathrm{HSB}}=5 \times 10^{11}$. Yet the range of mass-toluminosity ratios for the two samples of galaxies is quite different. The median $M_{\mathrm{HI}} / L_{\mathrm{B}}$ value for the LSB galaxies is $2.8 M_{\odot} / L_{\odot, \mathrm{B}}$ while $\left\langle M_{\mathrm{HI}} / L_{\mathrm{B}}\right\rangle_{\text {median }}^{\mathrm{HSB}}=0.4 M_{\odot} / L_{\odot, \mathrm{B}}$. The gasto-dynamical mass ratio shows a similar, albeit smaller, trend with $\left\langle M_{\mathrm{HI}} / M_{\mathrm{dyn}}\right\rangle_{\text {median }}^{\mathrm{LSB}}=0.01$ and $\left\langle M_{\mathrm{HI}} / M_{\mathrm{dyn}}\right\rangle_{\text {median }}^{\mathrm{HSB}}=0.006$.

The higher H I mass-to-luminosity ratios $\left(M_{\mathrm{HI}} / L_{\mathrm{B}}\right)$ found for the LSB galaxies may be a strong indicator that the LSB systems have evolved differently from their HSB counterparts. This idea is by no means a new one - that LSB galaxies in general have high H I mass-to-luminosity ratios is well established. In 1982, Romanishin et al. first reported that galaxies of lower surface brightness have $M_{\mathrm{HI}} / L_{\mathrm{B}}$ values approximately 2.4 times higher than for similar HSB samples. This trend has continued for all $\mathrm{H}$ I studies undertaken of these diffuse systems (e.g. de Blok et al. 1996; O'Neil et al. 2000; Schombert et al. 2001; Burkholder et al. 2001) indicating that LSB galaxies are less evolved, less efficient in their star formation, or have otherwise undergone a different evolutionary scenario then their 

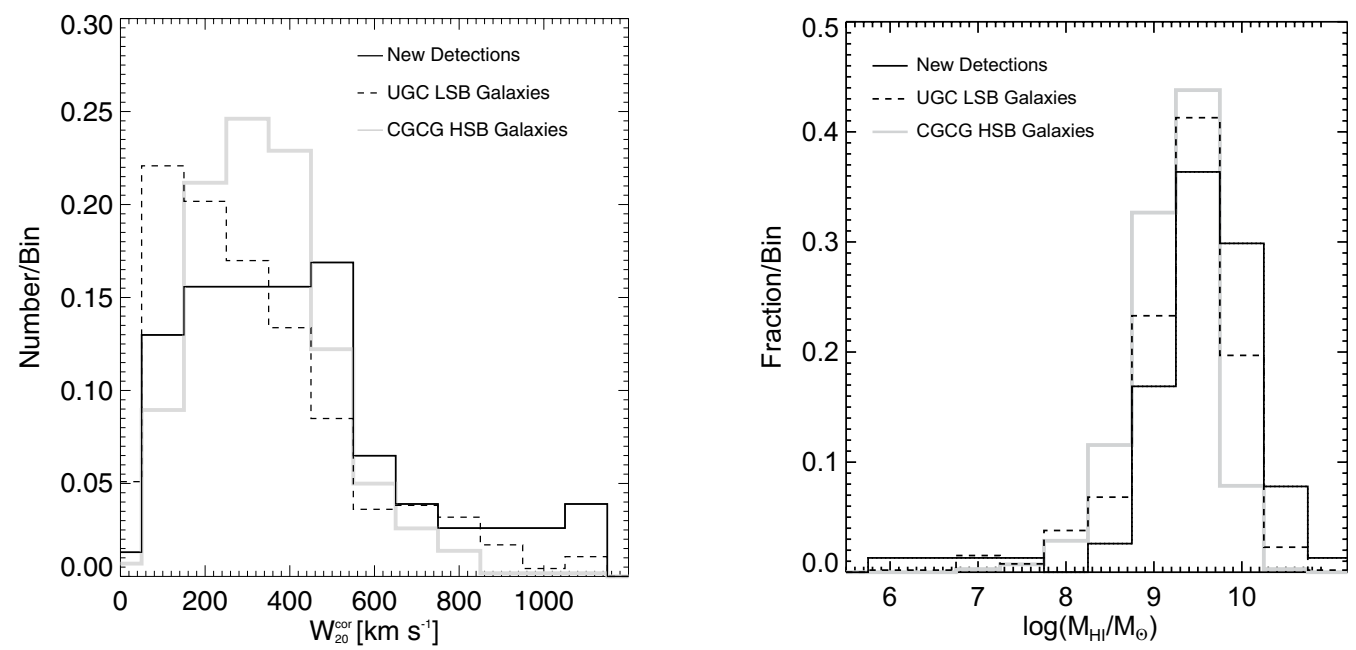

Fig. 3. The fractional distribution of velocity widths (at $20 \%$ of the peak, and corrected for inclination, left) and H I masses (right) for all the galaxies in this survey. In both plots, the new detections are shown by the solid black line while the results from all LSB UGC galaxies in this study are shown as the dashed line. Additionally, the results from a similar H I survey done by Giovanelli \& Haynes (1993) of HSB CGCG galaxies is shown by the thick gray line. In the plot on the left, the inclination correction applied is $\operatorname{simply} W_{20}^{\text {corr }}=W_{20} / \sin (i)$. To avoid over-correction, any inclination less than $30^{\circ}$ has been set to $30^{\circ}$ for the purpose of this correction. Note that the extremely high values of $W_{20}^{\text {corr }}$ may be due to an underestimate of the galaxy's inclination (see Fig. 4).

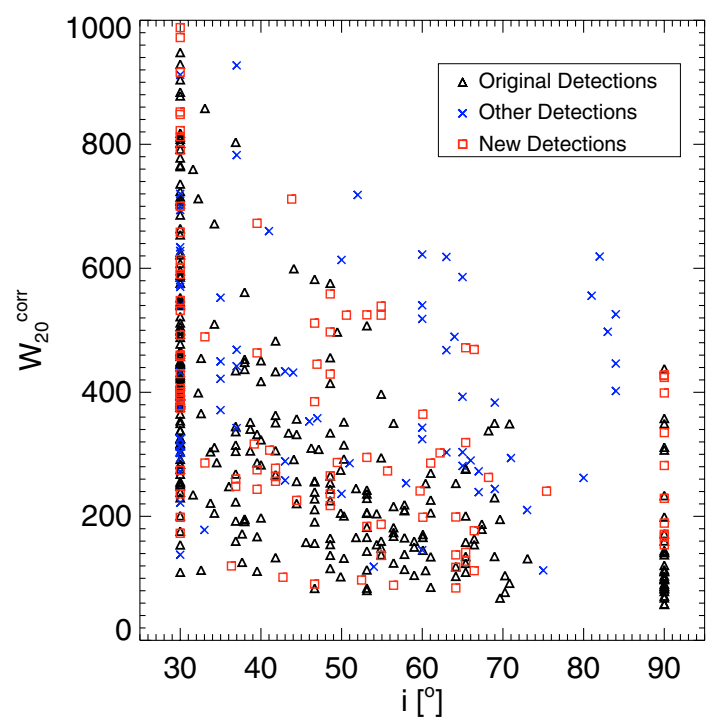

Fig. 4. The inclination of each LSB galaxy in our survey versus its inclination-corrected velocity width. From this plot it can be seen that the extremely high values of $W_{20}^{\text {corr }}$ are found when the inclinations are the lowest. This indicates that the inclination in those cases may be underestimated, artificially increasing the values for $W_{20}^{\text {corr }}$. The inclination plotted is that given by the UGC catalog, except in the cases where $i<30^{\circ}$. To avoid over-correction, any inclination less than $30^{\circ}$ has been set to $30^{\circ}$ for the purpose of the velocity width correction.

HSB counterparts (see, e.g., the modeling by Boissier et al. 2003).

However, this brings us to a logical paradox that was first explored by de Blok \& McGaugh (1998). Our calculations of dynamical mass (e.g. $v^{2} R$ ) use the tabulated UGC diameter as the basis for calculating $R$. In general this diameter corresponds to an isophotal limit of $25 \mathrm{mag} \operatorname{arcsec}^{-2}$ (Cornell et al. 1987). For a typical HSB galaxies with $\mu_{\mathrm{B}}(0)=21.5$, this isophotal

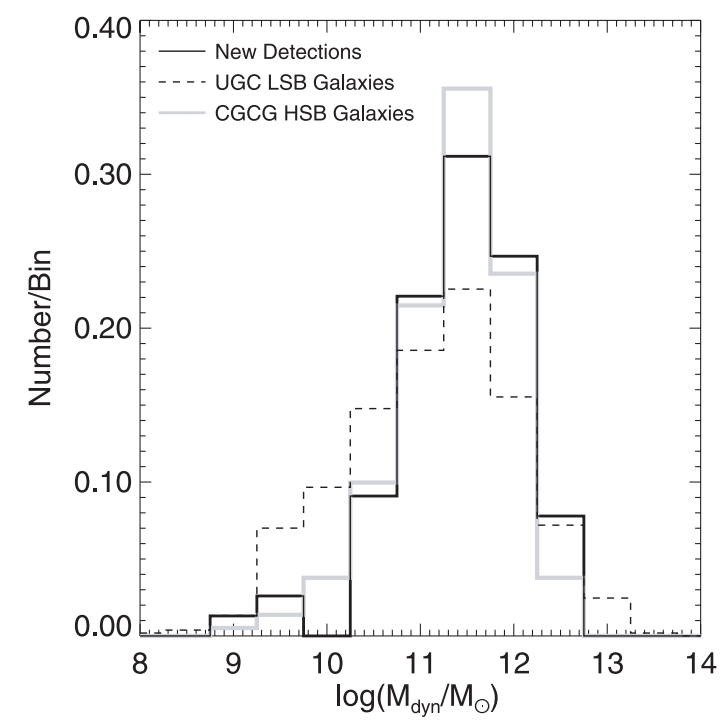

Fig. 5. The fractional distribution of dynamical mass for all the galaxies in this survey. In both plots, the new detections are shown by the solid black line while the results from all LSB UGC galaxies in this study are shown as the dashed line. Additionally, the results from a similar survey done by Giovanelli \& Haynes (1993) looking for H I in the HSB galaxies of the CGCG is shown by the thick gray line.

radius corresponds to $\sim 3$ optical disk scale lengths. However, for a typical LSB galaxy with $\mu_{\mathrm{B}}(0)=23.5$, this isophotal radius is only $1-2$ scale lengths. This means that for given values of circular velocity, redshift, and apparent diameter, an LSB will have a dynamical mass which is systematically underestimated compared to that of an HSB galaxy. We estimate that this error could be as high as a factor of two or more. Therefore, if a constant number of scale lengths were used in the determination of the radius, the fractional $\mathrm{H}$ I content of LSB disks would actually be lower than that of HSB disks. This is another way 

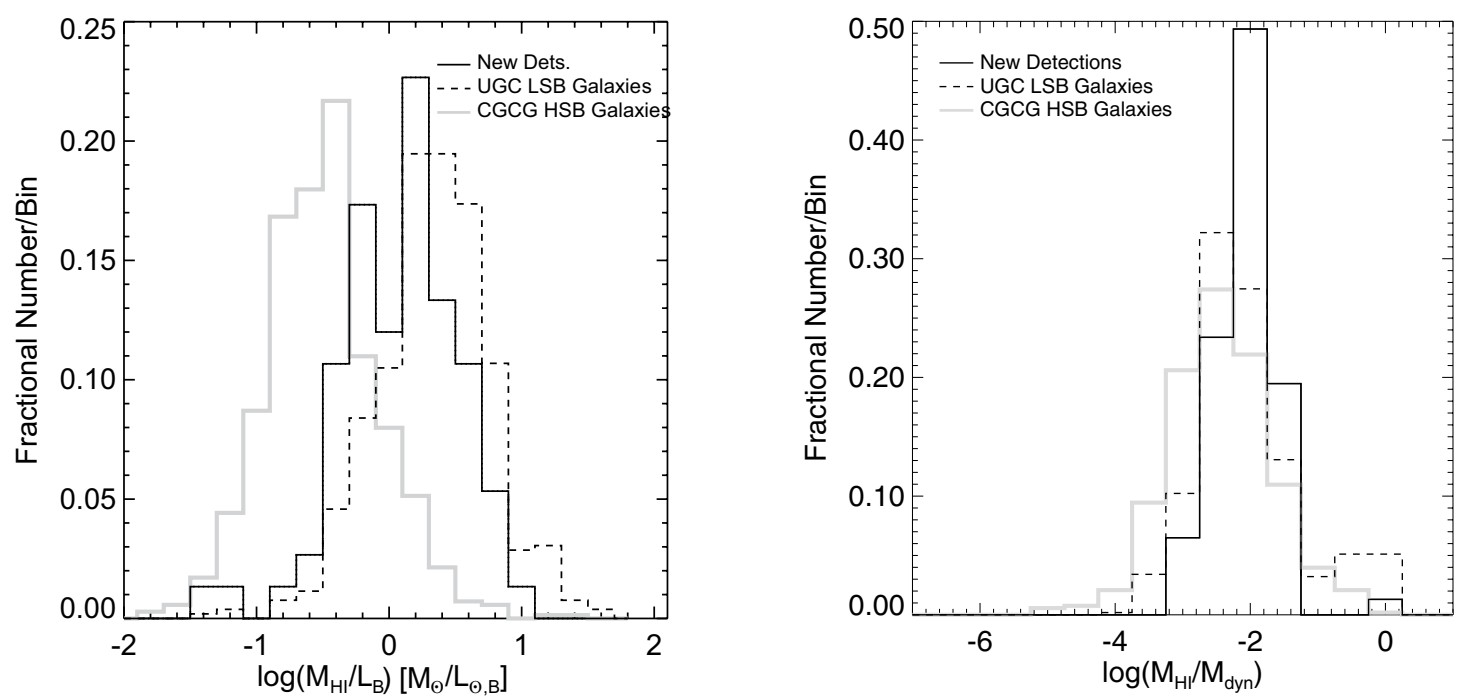

Fig. 6. The fractional cumulative distribution of HI mass-to-luminosity ratio (left) and HI mass-to-dynamical mass (right) for the UGC LSB galaxies. In both plots, the new detections are shown by the solid black line while the results from all LSB UGC galaxies in this study are shown as the dashed line. Additionally, the results from a similar survey done by Giovanelli \& Haynes (1993) looking for H I in the HSB galaxies of the CGCG is shown by the thick gray line.

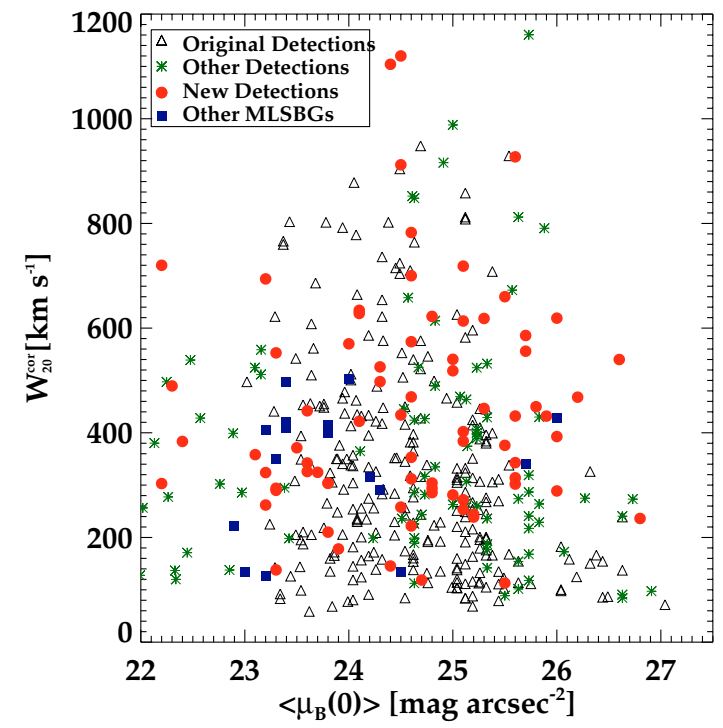

Fig. 7. Surface brightness versus (inclination corrected) velocity widths both for all the galaxies in our LSB UGC sample and for the 16 massive LSB galaxies with cataloged H I properties (Matthews et al. 2001; Sprayberry et al. 1995). The inclination correction applied is simply $W_{20}^{\text {corr }}=W_{20} / \sin (i)$. To avoid over-correction, any inclination less than $30^{\circ}$ has been set to $30^{\circ}$ for the purpose of this correction. Note that the extremely high values of $W_{20}^{\text {corr }}$ may be due to an underestimate of the galaxy's inclination (see Fig. 4).

of saying that LSB disks must have a lower baryonic mass fraction than HSB disks. Similar conclusions, based on mass modeling of LSB galaxies with rotation curves, have been reached by McGaugh \& de Blok (1998) and Pickering et al. (1999). This lower baryonic mass fraction could result in different star formation histories between LSB and HSB disks.

Indeed, Fig. 8a shows a curious correlation between $\left\langle\mu_{\mathrm{B}}\right\rangle$ and $M_{\mathrm{HI}} / L_{\mathrm{B}}$, similar to that found by, e.g. Burkholder et al. (2001) - an increasing H I mass-to-luminosity ratio with decreasing surface brightness. Although there is a large scatter, the trend seen in this figure is significant. In particular, the mean value of $M_{\mathrm{HI}} / L_{\mathrm{B}}$ for $\left\langle\mu_{\mathrm{B}}\right\rangle=23.0,24.0$ and 25.0 is $0.24,0.37$, and 0.55 , respectively. To first order, these trends are physically reasonable. For instance, a purely gaseous disk sitting in some dark matter potential will have very low optical surface brightness and a very high gas to star ratio. However, that simplistic scenario would also predict a correlation between fractional gas content and surface brightness. That is, as star formation begins to consume the gas and thus elevate the surface brightness of the disk, the fractional $\mathrm{H}$ I content of the potential would diminish. One should then observe the lowest values of $\left\langle\mu_{\mathrm{B}}\right\rangle$ corresponding to the highest values of $M_{\mathrm{HI}} / M_{\mathrm{dyn}}$. However, this is clearly not seen as these two parameters are essentially uncorrelated (Fig. 8b).

Thus, while the average gas-to-light ratio of galaxies appears to increase considerably with decreasing surface brightness, the average gas-to-total mass ratio does not show any increase. Indeed, if the dynamical masses of the lower surface brightness galaxies are underestimated, as discussed above, using accurate measurements of $M_{\mathrm{dyn}}$ could result in the inversea decrease of $M_{\mathrm{HI}} / M_{\mathrm{dyn}}$ with $\left\langle\mu_{\mathrm{B}}\right\rangle$. If the only factor causing the $M_{\mathrm{HI}} / L_{\mathrm{B}}-\mu_{\mathrm{B}}$ correlation were a slower or less efficient evolution of LSB systems, the correlation should be equally evident between $\left\langle\mu_{\mathrm{B}}\right\rangle$ and both $M_{\mathrm{HI}} / M_{\mathrm{dyn}}$ and $M_{\mathrm{HI}} / L_{\mathrm{B}}$. As this is not the case, additional possibilities for reducing the luminosity of a galaxy with surface brightness while keeping the gas-to-total mass ratio the same must be considered. This is where the physical meaning of surface brightness comes into play, as surface brightness is the convolution of the mean (blue) luminosity of the stellar population and the average separation between the stars. One explanation of these trends is that the process of star formation in LSB disks is sufficiently different than that seen in HSB disks so as to reduce the natural coupling between increased blue luminosity and increased blue surface 

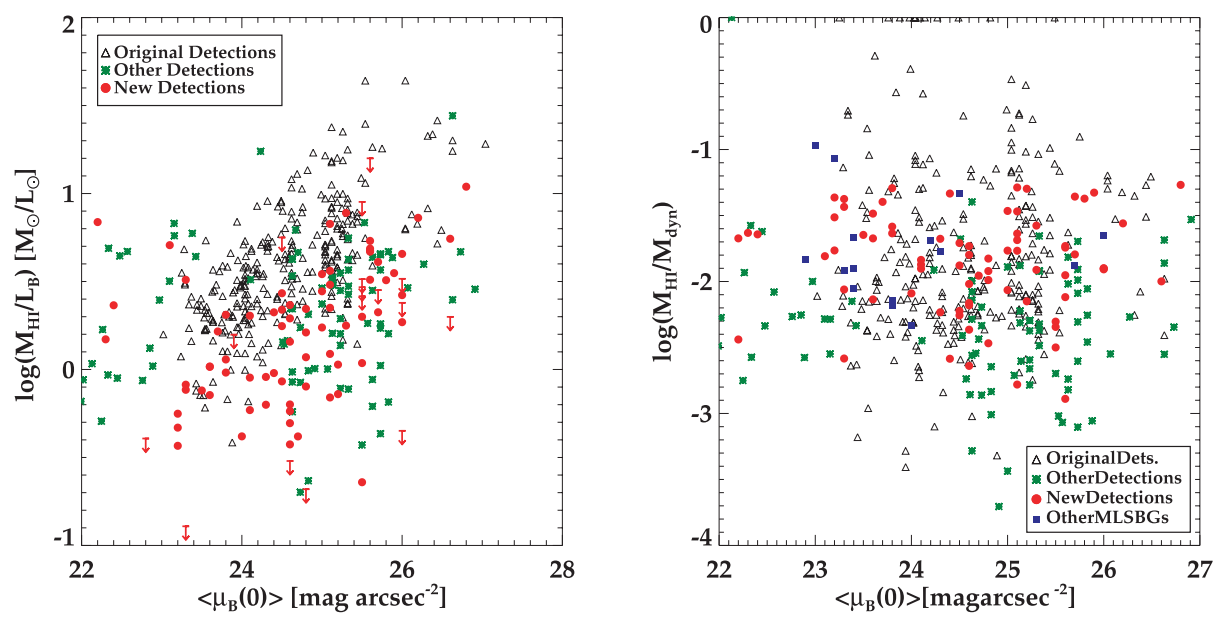

Fig. 8. Surface brightness versus $M_{\mathrm{HI}} / L_{\mathrm{B}}$ (left) and $M_{\mathrm{HI}} / M_{\mathrm{dyn}}$ (right) for all the galaxies in our LSB UGC sample, as well as the 16 massive LSB galaxies with published H I properties (Matthews et al. 2001). Those objects which were observed in our survey which have published velocities but which were not detected are given upper limits to their flux equal to $3 \sigma \times\left\langle W_{20}\right\rangle$, where $\left\langle W_{20}\right\rangle=300 \mathrm{~km} \mathrm{~s}^{-1}$. These objects are shown as arrows in the left plot.
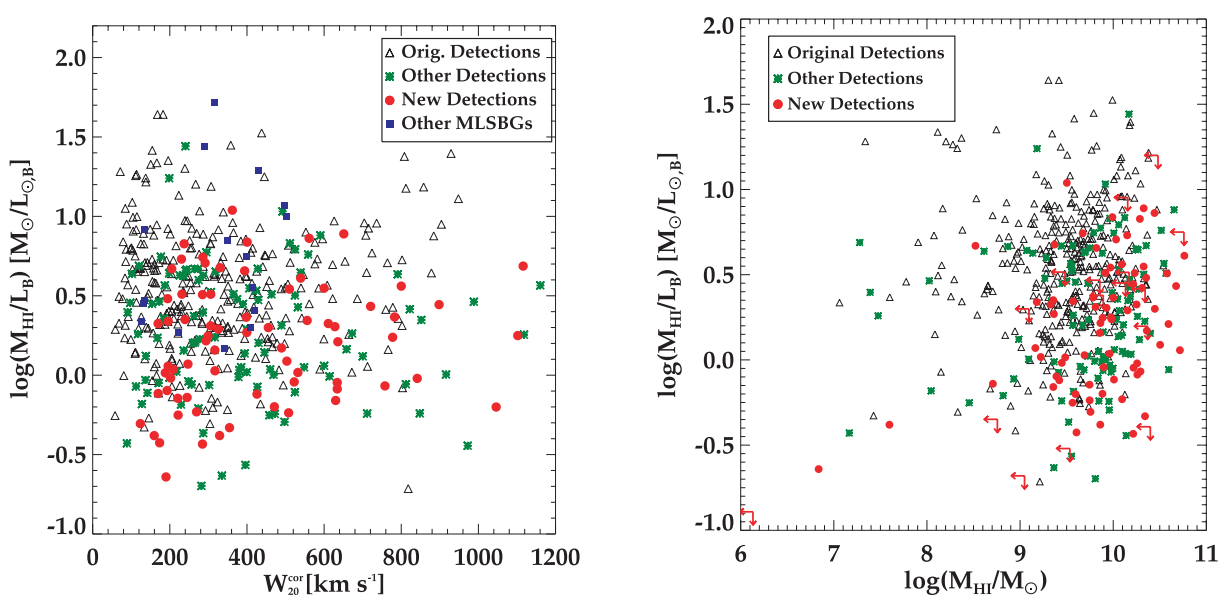

Fig. 9. Velocity width (corrected for inclination) (left) and H I gas mass (right) versus $M_{\mathrm{HI}} / L_{\mathrm{B}}$ for all the galaxies in our LSB UGC sample. Those objects which were observed in our survey which have published velocities but which were not detected are given upper limits to their flux equal to $3 \sigma \times\left\langle W_{20}\right\rangle$, where $\left\langle W_{20}\right\rangle=300 \mathrm{~km} \mathrm{~s}^{-1}$. These objects are shown as arrows in the plots. In the plot on the left, the inclination correction applied is simply $W_{20}^{\text {corr }}=W_{20} / \sin (i)$. To avoid over-correction, any inclination less than $30^{\circ}$ has been set to $30^{\circ}$ for the purpose of this correction. Note that the extremely high values of $W_{20}^{\text {corr }}$ may be due to an underestimate of the galaxy's inclination (see Fig. 4). In addition, the extremely high values for $M_{\mathrm{HI}} / L_{\mathrm{B}}$ are likely due to underestimates of $L_{\mathrm{B}}$ in the UGC catalog, a result of the galaxies' LSB nature.

brightness. This can be accomplished (as has been suggested earlier by O'Neil et al. 2000 and Bothun et al. 1997) if star formation in LSB disks occurs in a lower density gas environment which produces a larger than average separation between newly formed stars.

\section{Conclusions and discussion}

Our survey, undertaken with both the Arecibo and Nançay radio telescopes, has resulted in the determination of H I properties and redshifts for 81 UGC LSB galaxies, 38 of which can be classified as massive "Malin 1 cousins". This has increased the number of known massive LSB galaxies by a factor of 3 . Combining our results with all previous $21-\mathrm{cm}$ observations of the Bothun et al. (1985) UGC LSB galaxy list results in a final catalog of 526 LSB galaxies, with properties ranging across the known gamut of disk system properties, including numerous extremely gas-rich galaxies with $M_{\mathrm{HI}} / L_{\mathrm{B}} \geq 10 M_{\odot} / L_{\odot, \mathrm{B}}$. Comparing the properties of these galaxies with those found in similar H I surveys of HSB galaxies surveys shows the curious fact that while the mean $M_{\mathrm{HI}} / L_{\mathrm{B}}$ value increases with decreasing surface brightness, the $M_{\mathrm{HI}} / M_{\mathrm{dyn}}$ ratio does not. This raises the (currently unanswered) question of whether or not star formation is significantly affected by the low density environment found within LSB galaxies.

With this catalog in hand, we can now ask the question What do we gain by continuing to look for and study massive LSB systems? The answer to this question is (at least) threefold.

First, as massive LSB galaxies are significant repositories of baryonic (and dark) matter (Pickering et al. 1997, 1999; Walsh et al. 1997), determining both the number density and distribution of these objects could provide considerable insight 

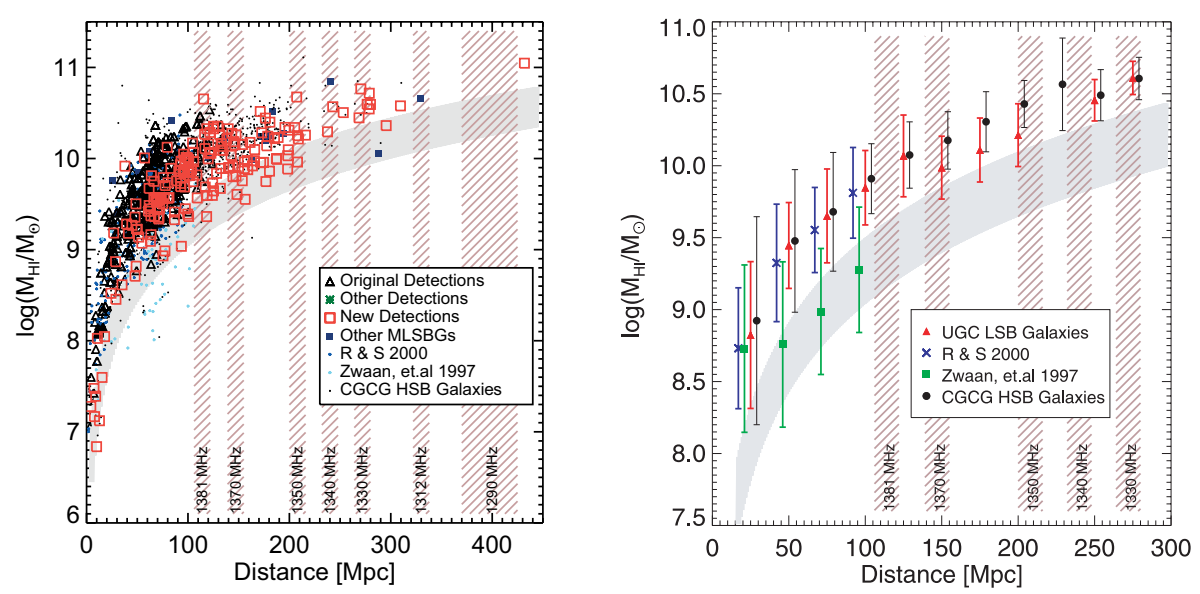

Fig. 10. Distance $\left(\frac{\text { velocity }}{H_{0}}\right)$ plotted against total H I mass for all the galaxies in the total UGC LSB galaxy discussed herein (Sect. 2). For comparison, objects found in the two Arecibo blind H I surveys (Rosenberg \& Schneider 2000; Zwaan et al. 1997) and the Giovanelli \& Haynes (1993) CGCG survey are also plotted. The gray line on the plots indicates the approximate survey limit for our observations, where the line thickness represents the varying sensitivities and velocity widths found herein. Finally, the diagonal lines show the primary regions affected by RFI. On the left the individual data points are shown, and the 16 other massive LSB galaxies with published H I masses (from Matthews et al. 2001 and Sprayberry et al. 1995) are also plotted. On the right, the mean values for the various surveys are plotted, with $25 \mathrm{Mpc}$ bins. Note the data points in each bin are offset in the in the $x$-direction for clarity - the points should lie over the red symbols.
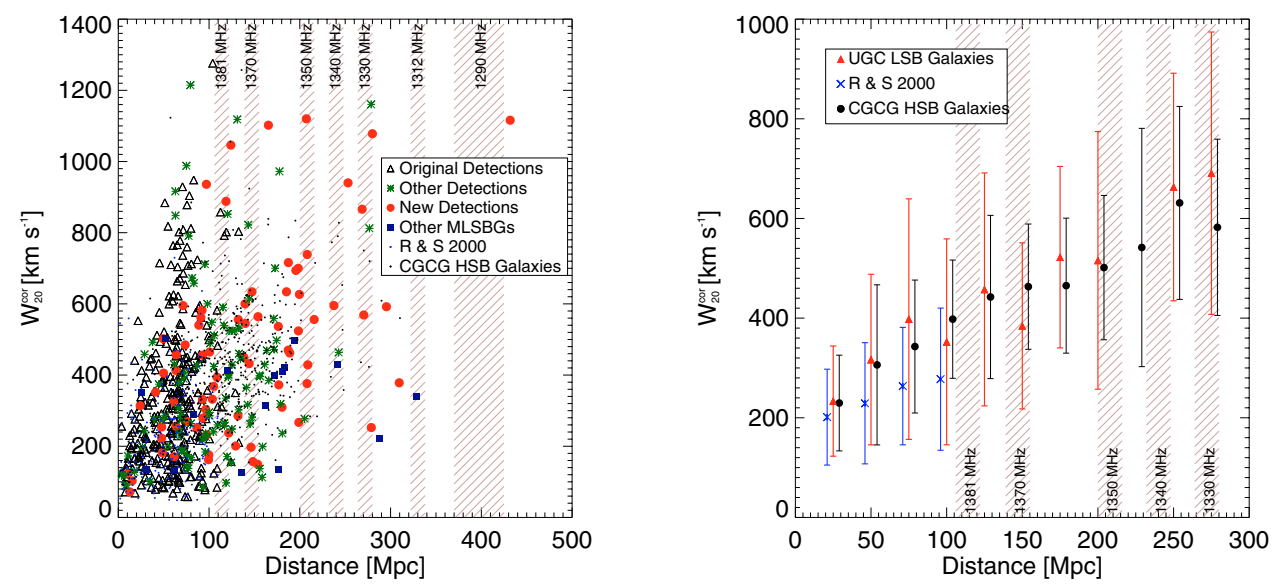

Fig. 11. Distance $\left(\frac{\text { velocity }}{H_{0}}\right)$ plotted against velocity width (corrected for inclination) for all the UGC LSB galaxies discussed herein (Sect. 2). For comparison, objects found in the Rosenberg \& Schneider (2000) Arecibo blind H I survey and the Giovanelli \& Haynes (1993) CGCG survey are also plotted. (The Zwaan et al. 1997 survey did not publish velocity widths.) Again, the diagonal lines show the primary regions affected by RFI. On the left the individual data points are shown, and the 16 other massive LSB galaxies with published H I masses (from Matthews et al. 2001 and Sprayberry et al. 1995) are also plotted. On the right, the mean values for the various surveys are plotted, with 25 Mpc bins. Note the data points are offset in the in the $x$-direction for clarity - the points should lie over the red symbols. In the plot on the left, the inclination correction applied is simply $W_{20}^{\text {corr }}=W_{20} / \sin (i)$. To avoid over-correction, any inclination less than $30^{\circ}$ has been set to $30^{\circ}$ for the purpose of this correction. Note that the extremely high values of $W_{20}^{\text {corr }}$ may be due to an underestimate of the galaxy's inclination (see Fig. 4).

into the overall distribution of matter in the Universe. While the survey described herein cannot determine the number density of massive LSB galaxies, it does show that there are more of these objects in the Universe than previously known, and determining the density and distribution of these massive objects will allow for a better understanding of the cosmological distribution of mass throughout the universe.

In this respect, it is vital here to point out the effect surveys such as this one have on determining the LSB galaxy contribution to baryon density. Because of their perceived morphology on photographic plates lacking the sensitivity to show their outer disks, and their previous lack of detection in $\mathrm{HI}$, all of the massive LSB galaxies detected in our catalog were previously believed to be dwarf systems with little to no H I mass. As a result, our detections are not merely adding a few massive LSB galaxies to the number counts, but moving galaxies from being listed as extremely low mass to extremely high mass systems, and thus shifting the distribution of masses in the luminosity function.

Second, and equally important, massive LSB galaxies provide a unique insight into galaxy evolution studies as a whole. Because massive LSB galaxies reside at an extreme end of 
the mass and density distribution of galaxies, any model that wishes to describe galaxy evolution must be able to account for them. And, as massive LSB galaxies appear to be undeveloped in at least some aspects, when compared to HSB galaxies (e.g., their higher than average $M_{\mathrm{HI}} / L_{\mathrm{B}}$ ratios and often sparse H- $\alpha$ emission - Pickering et al. 1997), studies of massive LSB galaxies, and of LSB galaxies in general, offer a look into a different aspect of galaxy formation than those of HSB galaxies.

Third, it has been shown that if LSB galaxies are as common as HSB galaxies, then they may be one of the primary causes for Lyman- $\alpha$ absorbers in the local Universe (Linder 1998, 2000). As a result, knowing the density of massive LSB galaxies could lead to a allow for considerably better understanding of the nature of Lyman- $\alpha$ absorption lines. This would allow us to construct a more complete picture of the large-scale gas distribution in the Universe, as well as providing insight into galaxy formation processes.

At this point it is clear that we do not yet have an answer regarding the number density of massive LSB galaxies, as the blind surveys done to date (both optical, near-infrared and $\mathrm{HI}$ ) have not yet probed to sufficient sensitivity to allow this question to be answered. Clearly there is a dearth of such objects in the $z<0.01$ Universe, just as there is a shortage of all massive galaxies in that region. What is significant, though, is that as increasingly sensitive instruments become available, the number of LSB and massive LSB galaxies known continues to increase. (Notably, in a survey similar to ours, Schwortz et al. (2004) found another 9 massive LSB galaxies.) However, as the nature of pointed surveys such as the one described herein prevents them from providing an unbiased view into the number density of various galaxy types, we can only use these surveys to get a glimpse into what may be out there. The true number density of massive LSB galaxies will likely not be known until an extremely sensitive, large scale survey is undertaken, such as may be possible with the Arecibo $L$-band Feed Array (ALFA) or, ultimately, the Square Kilometre Array (SKA).

Acknowledgements. The Arecibo Observatory is part of the National Astronomy and Ionosphere Center, which is operated by Cornell University under a cooperative agreement with the National Science Foundation. The Nançay radio astronomy station, which is the Unite Scientifique de Nançay of the Observatoire de Paris, is associated with the French Centre National de Recherche Scientifique (CNRS) as USR B704, and acknowledges the financial support of the Région Centre as well as of the European Union. This research has made use of the Lyon-Meudon Extragalactic Database (LEDA), recently incorporated in HyperLeda, and of the NASA/IPAC Extragalactic Database (NED) which is operated by the Jet Propulsion Laboratory, California Institute of Technology, under contract with the National Aeronautics and Space Administration.

\section{References}

Bell, E., Bower, R., de Jong, R., Hereld, M., \& Rauscher, B. 1999, MNRAS, 30, L55

Bell, E., Barnaby, D., Bower, R. G., et al. 2000, MNRAS, 312, 470
Bell, E., McIntosh, D., Katz, N., \& Weinberg, M. 2003, ApJS, 149, 289

Bergmann, M., Jørgensen, I., \& Hill, G. 2003, AJ, 12, 116

Blanton, M., Hogg, D. W., Bahcall, N. A., et al. 2003, ApJ, 592, 819

Boissier, S., Monnier Ragaigne, D., Prantzos, N., et al., MNRAS, 343, 643

de Blok, W. J. G., \& McGaugh, S. 1998, ApJ, 508, 132

de Blok, W. J. G., van der Hulst, J. M., \& Bothun, G. D. 1995, MNRAS, 274, 235

de Blok, W. J. G., McGaugh, S., \& van der Hulst, J. M. 1996, MNRAS, 283, 18

Bothun, G. D. 2003, in The IGM/Galaxy Connection: The Distribution of Baryons at $z=0$, ed. J. Rosenburg, \& M. Putman, ASSL Conf. Proc., 281, 11

Bothun, G. D., Beers, T. C., Mould, J. R., \& Huchra, J. P. 1985, AJ, 90,2487

Bothun, G. D., Impey, C., \& McGaugh, S. 1997, PASP, 109, 745

Bottinelli, L., Gouguenheim, L., Fouqué, P., \& Paturel, G. 1990, A\&AS, 82, 391

Briggs, F. 1997, ApJ, 484, 618

Burkholder, V., Impey, C., \& Sprayberry, D. 2001, AJ, 122, 2318

Cabanela, J. E., \& Dickey, J. M. 2002, AJ, 124, 78

Colless, M., Ellis, R., Bland-Hawthorn, J., et al. 1999, in Looking Deep in the Southern Sky, ed. F. Morganti, \& W. Couch (Berlin: Springer-Verlag), 9

Cornell, M. E., Aaronson, M., Bothun, G., \& Mould, J. 1987, ApJS, 64, 507

Cross, N., Driver, S. P., Couch, W., et al. 2001, MNRAS, 324, 825

Cross, N., Driver, S., Liske, J., et al. 2004, MNRAS, preprint

Davies, J. I., Phillipps, S., \& Disney, M. J. 1988, MNRAS, 231, 69

Dekel, A., \& Woo, J. 2003, MNRAS, 344, 1311

Falco, E. E., Kurtz, M. J., Geller, M. J., et al. 1999, PASP, 111, 438

Fouqué, P., Durand, N., Bottinelli, L., Gouguenheim, L., \& Paturel, G. 1990, A\&AS, 86, 473

Fukugita, M., Hogan, C. J., \& Peebles, P. J. E. 1998, ApJ, 503, 518

Gerritsen, J., \& de Blok, W. J. G. 1999, A\&A, 342, 655

Giovanelli, R., Avera, E., \& Karachentsev, I. 1997, AJ, 114, 122

Giovanelli, R., Haynes, M. P., da Costa, L. N., et al. 1997, ApJ, 477, L1

Giovanelli, R., \& Haynes, M. P. 1993, AJ, 105, 1271

Heiles, C. 2001, Arecibo Technical and Operations Memo Series 2001-01http://www.naic.edu/ astro/aotms/ performance.shtml

Huchra, J., Vogeley, M., \& Geller, M. 1999, ApJS, 121, 287

Impey, C., \& Bothun, G. 1997, ARA\&A, 35, 267

Jimenez, R., Padoan, P., Matteucci, F., \& Heavens, A. 1998, MNRAS, 299, 123

de Jong, R., \& Lacey, C. 2000, ApJ, 545, 781

Kilborn, V. K., Webster, R. L., Staveley-Smith, L., et al. 2002, AJ, 124,690

Knezek, P. 1999, PASA, 16, 60

Koribalski, B. S. 2002, in Seeing Through the Dust: The Detection of $\mathrm{HI}$ and the Exploration of the ISM in Galaxies, ed. A. R. Taylor, T. L. Landecker, \& A. G. Willis

Kraan-Korteweg, R., van Driel, W., Briggs, F., Binggeli, B., \& Mostefaoi, T. 1999, A\&AS, 135, 255

Linder, S. 1998, ApJ, 495, L637

Linder, S. 2000, ApJ, 529, L644

Markze, R., Huchra, J., \& Geller, M. 1996, AJ, 112, 1803

Matthews, L. D., \& van Driel, W. 2000, A\&AS, 143, 421

Matthews, L. D., van Driel, W., \& Monnier-Ragaigne, D. 2001, A\&A, 365,1

Mathewson, D., \& Ford, V. 1996, ApJS, 107, 97 
McGaugh, S. 1996, MNRAS, 280, 337

McGaugh, S., \& de Blok, E. 1998, in Galactic Halos: A UC Santa Cruz Workshop, ed. D. Zaritsky, ASP Conf. Ser., 136, 210

Mobasher, B., Colless, M., Carter, D., et al. 2003, ApJ, 587, 605

Monnier Ragaigne, D., van Driel, W., Schneider, S. E., Balkowski, C., \& Jarrett, T. H. 2003, A\&A, 408, 465

Nilson, P. 1973, Uppsala General Catalogue of Galaxies, Uppsala Astron. Obs. Ann., 6 (UGC)

O'Neil, K. 2004, AJ, preprint

O’Neil, K., \& Bothun, G. 2000, ApJ, 529, 811

O’Neil, K., Bothun, G., Schombert, J., Cornell, M. E., \& Impey, C. D. 1997, AJ, 114, 2448

O'Neil, K., Bothun, G., \& Schombert, J. 2000, AJ, 119, 136

O’Neil, K., Andreon, S., \& Cuillandre, J.-C. 2003, A\&A, 399, L35

Pickering, T. E., Impey, C., van Gorkom, J. H., \& Bothun, G. D. 1997, AJ, 114, 1858

Pickering, T. E., van Gorkom, J. H., Impey, C., \& Quillen, A. 1999, AJ, 118, 765

Romanishin, W., Krumm, N., Salpeter, E., et al. 1982, ApJ, 263, 94

Rosenberg, J. L., \& Schneider, S. E. 2000, ApJS, 130, 177

Sabatini, S., Davies, J., Scaramella, R., et al. 2003, MNRAS, 341, 981

Schwortz, A. C., Schneider, S. E., \& van Driel, W. 2004, in preparation

Schombert, J., McGaugh, S., \& Eder, J. 2001, AJ, 121, 2420
Shull, J. M. 2003, in The IGM/Galaxy Connection: The Distribution of Baryons at $z=0$, ed. J. Rosenburg, \& M. Putman, ASSL Conf. Proc., 281, 1

Sorar, E. 1994, Ph.D. Dissertation, Pittsburgh University

Spergel, D. N., Verde, L., Peiris, H. V., et al. 2003, ApJS, 148, 175

Sprayberry, D., Impey, C. D., Bothun, G. D., \& Irwin, M. J. 1995, AJ, 10,558

Schwartzenberg, J. M., Phillipps, S., Smith, R. M., Couch, W. J., \& Boyle, B. J. 1995, MNRAS, 275, 121

Strauss, M., Weinberg, D. H., Lupton, R. H., et al. 2002, AJ, 124, 1810

van Driel, W., Gérard, E., \& Pezzani, J. 1997, The FORT project: renovating the Nançay radio telescope. In High Sensitivity Radio Astronomy, ed. N. Jackson, \& R. J. Davies (Cambridge: Cambridge Univ. Press), 229

Walsh, W., Staveley-Smith, L., \& Osterloo, T. 1997, AJ, 113, 1851

Zwaan, M., Staveley-Smith, L., Koribalski, B. S., et al. 2003, AJ, 125, 2842

Zwaan, M., Briggs, F., Sprayberry, D., \& Sorar, E. 1997, ApJ, 490, 173

Zwicky, F., Herzog, E., Wild, P., et al. 1961-1966, Catalogue of Galaxies and of Clusters of Galaxies, Publ. California Inst. of Technology, Vols. 1-6 
K. O'Neil et al.: Tripling the number of massive LSBGs, Online Material p 1

\section{Online Material}




\section{Appendix A: Notes on individual galaxies}

a. UGC 00134: this detection is likely UGC 00132, which at $v_{\mathrm{HEL}}=1666 \mathrm{~km} \mathrm{~s}^{-1}$ and 4.0 away is within the Nançay beam.

b. UGC 02641: ARK 099, at $v_{\mathrm{HEL}}=7080 \mathrm{~km} \mathrm{~s}^{-1}$ and 1.4 away, is within the Arecibo beam. Both UGC 02641 and ARK 099 are of late (S?) morphological type and neither have published H I spectra. It is likely that both galaxies contribute to the observed H I spectra.

c. UGC 03225, UGC 3790: these objects are listed in NED as galaxy pairs.

d. UGC 04496: NRGb 004.06, at $v_{\mathrm{HEL}}=8730 \mathrm{~km} \mathrm{~s}^{-1}$ and 0 .'1 away, is within the Arecibo beam. It is likely that both galaxies lie within the observed H I spectra.

e. UGC 05769, UGC 05770 Both of these galaxies lie within the observed H I spectra.

f. UGC 06947: CGCG 127-113, at $v_{\mathrm{HEL}}=9106 \mathrm{~km} \mathrm{~s}^{-1}$ and 4.2 away, is still within the first Arecibo side lobe. However, CGCG 127-113 is classified as an E0 galaxy and so is unlikely to contribute much (if any) gas to the H I spectra measured for UGC 06947.

g. UGC 07437: this object is a galaxy pair with one galaxy at $v_{\mathrm{HEL}}=19669 \mathrm{~km} \mathrm{~s}^{-1}$ and the other at $19319 \mathrm{~km} \mathrm{~s}^{-1}$. It is doubtful that the smaller member of the pair (UGC 07437-01) is contributing significantly to the observed spectra, though, as it is classified as an E galaxy while UGC 07437 is an Sbc galaxy.

h. UGC 07438: NGC 4308, at $v_{\mathrm{HEL}}=624 \mathrm{~km} \mathrm{~s}^{-1}$ and 5' 1 away, could lie within the first side lobe of the Arecibo beam. NGC 4308 is classified as an E galaxy, though, and so is likely not contributing significantly to the total H I gas found. i. UGC 08637: UGC 08637 is listed as a galaxy pair within NED. However, no velocity is given for its companion and no evidence is visible within UGC 08637's spectra for contamination from another galaxy.

j. UGC 09010: UGC 09010 has two companions which could be contaminating the observed spectra - UGC 09012 at $v_{\text {HEL }}=7483 \mathrm{~km} \mathrm{~s}^{-1}$ and 3'2 away and MCG $+05-33-050$ at $v_{\mathrm{HEL}}=7628 \mathrm{~km} \mathrm{~s}^{-1}$ and 4.1 away. Both companion galaxies fall within the Arecibo beam and first side lobe. MCG +05-33-050 is classified as a S0 galaxy and is not likely contributing significantly to the observed H I flux. UGC 09012, though, is a large, bright, spiral galaxy which probably has considerably contaminated the observed flux.

k. UGC 09238: This galaxy has a companion galaxy, UGC 09235 at $v_{\text {HEL }}=2973 \mathrm{~km} \mathrm{~s}^{-1}$ and only 1.9 away, well within the Arecibo beam. It is likely that UGC 09235 is the reason for the apparently lopsided spectra seen for UGC 09238.

1. UGC 09513: UGC 09515 lies at $14011 \mathrm{~km} \mathrm{~s}^{-1}$ and 3'.0 from UGC 09513. UGC 09515 is listed as an S? galaxy, and could contain enough H I mass to considerably contaminate the H I flux found for UGC 09513.

m. UGC 10674: is not listed as either being a galaxy pair or having a companion within $50^{\prime}$. However, the spectra clearly shows two separate profiles.

n. UGC 11696: UGC 11694 is part of a galaxy group which also contains UGC $11696\left(v_{\mathrm{HEL}}=5018 \mathrm{~km} \mathrm{~s}^{-1}\right.$, distance $=6.3$, UGC $11700\left(v_{\mathrm{HEL}}=5036 \mathrm{~km} \mathrm{~s}^{-1}\right.$, distance $=11: 3)$, CGCG 426-015 $\left(v_{\mathrm{HEL}}=5031 \mathrm{~km} \mathrm{~s}^{-1}\right.$, distance=13.4), and UGC $11697\left(v_{\mathrm{HEL}}=5086\right.$, distance $=23 \cdot 4)$. As this galaxy was observed with the Nançay telescope, it is likely to be contaminated by one or more of UGC 11694's companions. 